\title{
Synthesizing Averaged Virtual Oscillator Dynamics to Control Inverters with an Output LCL Filter
}

\author{
M. Ali*, H. I. Nurdin and J. E. Fletcher \\ School of Electrical Engineering and Telecommunications, University of New South Wales, Australia \\ *m.ali@unsw.edu.au
}

\begin{abstract}
In commercial inverters, an LCL filter is considered an integral part to filter out the switching harmonics and generate a sinusoidal output voltage. The existing literature on the averaged virtual oscillator controller (VOC) dynamics is for current feedback before the output LCL filter that contains the switching harmonics or for inductive filters ignoring the effect of filter capacitance. In this work, a new version of averaged VOC dynamics is presented for islanded inverters with current feedback after the LCL filter thus avoiding the switching harmonics going into the VOC. The embedded droopcharacteristics within the averaged VOC dynamics are identified and a parameter design procedure is presented to regulate the output voltage magnitude and frequency according to the desired ac-performance specifications. Further, a power dispatch technique based on this newer version of averaged VOC dynamics is presented to simultaneously regulate both the active and reactive output power of two parallel-connected islanded inverters. The control laws are derived and a power security constraint is presented to determine the achievable power setpoint. Simulation results for load transients and power dispatch validate the proposed version of averaged VOC dynamics.
\end{abstract}

Index Terms-Virtual oscillator control, averaged model, LCL filter, inverter control, power dispatch, security constraint.

\section{INTRODUCTION}

Power systems are going through a transitional period where the conventional synchronous generators are being replaced by the renewable energy sources (RESs). The rapid integration of RESs into the power system has resulted in a need to investigate control techniques to stabilise and regulate the voltage and frequency for systems with low or zero inertia. Existing techniques for systems with RESs include droop control [1], [2], proportional resonant (PR) control [3], [4] and recently proposed virtual oscillator control (VOC) [5]-[7].

Virtual oscillator is a nonlinear Van der Pol oscillator that exhibits nearly sinusoidal oscillations in the steady-state. In contrast to existing inverter control techniques, virtual oscillator controller only requires the inverter output current as a feedback and regulates the output voltage (magnitude and frequency) according to the desired ac-performance specifications. In [5], an averaged VOC model is derived to explicitly identify the $P-V$ and $Q-\omega$ droop-characteristics embedded within the averaged dynamics of the virtual oscillator controller. Recent literature on the dispatchability of virtual oscillator controlled inverters can be found in [8]-[14].

The averaged VOC model presented in [5] is derived when there is a current feedback to the controller before the output filter. However, this model is not an accurate representation when there is a current feedback after the output LCL filter. In case of commercial inverters, an output LCL (or LC) filter is always considered an essential part to filter out the switching harmonics and improve the quality of output voltage. In this work, a new version of averaged VOC dynamics is presented that takes into account the LCL filter at the output of an inverter. In the proposed control scheme, the current is feedback after the LCL filter thus avoiding the switching harmonics going into the virtual oscillator controller. The contribution of this work is twofold. At first, an averaged VOC model is derived for an inverter with current feedback after the output LCL filter. The updated embedded droop-characteristics within the proposed averaged VOC dynamics are presented and corresponding equilibrium points are derived. A system's parameter design procedure has been presented to regulate the inverter's output voltage magnitude and frequency according to the desired ac-performance specifications. In order to validate the proposed averaged VOC dynamics, simulation results are presented for a number of scenarios including rise time, harmonics analysis, droop characteristics and load transients. It has been shown that for all the scenarios, the proposed averaged VOC model predicts the actual VOC (Van der Pol) dynamics accurately. A comparison with the previously reported averaged VOC model [5] is also presented. The previously derived averaged VOC dynamics [5] starts to differ more from the actual VOC dynamics (with current feedback after the LCL filter) for a large value of filter capacitance. This is due to the fact that a higher value of filter capacitance draws more current and results in a significant difference between the current before and after the filter.

The second contribution is the extension of power dispatch technique [9] to this new version of averaged VOC dynamics. The updated control laws are derived based on this new model to determine the control inputs corresponding to a particular power set-point. Further, an updated power security constraint is derived to determine the feasible power set-points. Using this constraint, it can be determined a-priori if a particular power set-point is achievable or not. Simulation results are presented for a number of power dispatch scenarios to validate the proposed power dispatch technique.

The rest of the paper is organised as follows. In Section [I]. an overall system description is presented. In Section III] an averaged VOC model is derived for inverters with current 


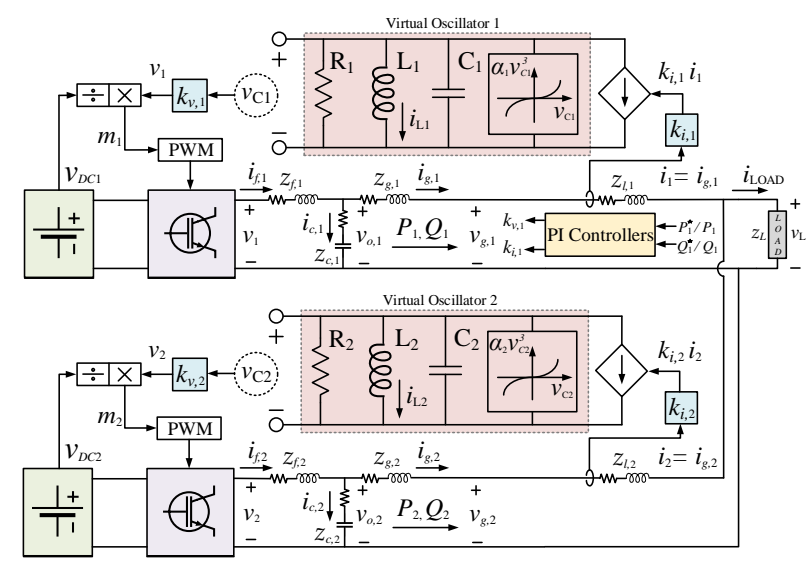

Figure 1. A overview of the overall system consisting of two parallelconnected virtual oscillator controlled inverters. The inverter 1 can dispatch power using the PI controllers while inverter 2 supplies the remaining power.

feedback after the LCL filter. In Section IV] a VOC parameter design procedure is discussed. In Section $[\mathrm{V}$, a power dispatch technique is presented. In Section VI] simulation results are presented. In Section VII, conclusion is drawn.

\section{SYSTEM DESCRIPTION}

The system considered consists of two virtual oscillator controlled inverters with current feedback after the output LCL filter. A complete system overview is shown in Fig. 11. The filter parameters are $z_{f}=R_{f}+j \omega^{*} L_{f}, z_{c}=R_{c}+\frac{1}{j \omega^{*} C_{f}}$ and $z_{g}=R_{g}+j \omega^{*} L_{g}$. The line impedance is represented as $z_{l}=R_{l}+j \omega^{*} L_{l}$. The inverters are connected to a common RL load $z_{L}=R_{L}+j \omega^{*} L_{L}$ through the point of common coupling (PCC). Moreover, inverter 1 can dispatch power by the use of two additional PI controllers.

\section{System ModelLing}

In this section, the VOC dynamics are presented. Further, an averaged VOC model is derived for inverters with current feedback after the output LCL filter. The dynamics of the PI controllers used for power dispatch are the same as in [9].

\section{A. Virtual Oscillator Controller}

A virtual oscillator consists of an LC harmonic oscillator with a fundamental frequency $\omega^{*}=1 / \sqrt{L C}$. It consists of a negative resistance element $R=-1 / \sigma$ and a nonlinear current source with a positive constant $\alpha$ as shown in Fig. 1 . The feedback current $i$ is scaled by the current feedback gain $k_{i}$ before entering the virtual oscillator. The $v_{C}$ denotes the capacitor voltage and $i_{L}$ denotes the inductor current. The capacitor voltage $v_{C}$ is scaled by the voltage scaling factor $k_{v}$ to generate the inverter output voltage $v$. The actual VOC dynamics are given by the following dynamic equations [5]:

$$
\begin{aligned}
\frac{d V}{d t} & =\frac{\epsilon \omega^{*}}{\sqrt{2}}\left(\sigma g(\sqrt{2} V \cos (\phi))-k_{v} k_{i} i\right) \cos (\phi), \\
\frac{d \phi}{d t} & =\omega^{*}-\frac{\epsilon \omega^{*}}{\sqrt{2} V}\left(\sigma g(\sqrt{2} V \cos (\phi))-k_{v} k_{i} i\right) \sin (\phi) .
\end{aligned}
$$

A detailed derivation and parametric description of the dynamical model for the inverter RMS output voltage magnitude $V$ and instantaneous phase angle $\phi=\omega t+\theta$ where $\theta$ is the phase offset with respect to $\omega t$, can be found in [5].
B. Averaged VOC Model for Inverters with Output LCL Filter

A new version of averaged VOC dynamics is presented for inverters with current feedback after the output LCL filter. A brief derivation is in Appendix A The proposed averaged VOC model is given by the following dynamic equations:

$$
\begin{gathered}
\frac{d}{d t} \bar{V}=\frac{\sigma}{2 C}\left(\bar{V}-\frac{\beta}{2} \bar{V}^{3}\right)-\frac{k_{v} k_{i}}{2 C}\left(\frac{C_{\alpha} \bar{P}}{\bar{V}}+\frac{S_{\alpha} \bar{Q}}{\bar{V}}+C_{\beta} \bar{V}\right), \\
\frac{d}{d t} \bar{\theta}=\omega^{*}-\omega+\frac{k_{v} k_{i}}{2 C}\left(\frac{C_{\alpha} \bar{Q}}{\bar{V}^{2}}-\frac{S_{\alpha} \bar{P}}{\bar{V}^{2}}-S_{\beta}\right),
\end{gathered}
$$

where $\beta=\frac{3 \alpha}{k_{v}^{2} \sigma}, \bar{V}$ denotes the averaged RMS output voltage magnitude and $\bar{\theta}$ denotes the averaged phase offset with respect to $\omega t$. The averaged active and reactive power are denoted by $\bar{P}$ and $\bar{Q}$, respectively. $C_{\alpha}, C_{\beta}, S_{\alpha}$ and $S_{\beta}$ are the impedance constants (as defined in Appendix A).

1) Voltage Regulation Characteristics: The equilibrium value for the averaged RMS voltage magnitude $\bar{V}_{e q}$ can be determined by setting the left hand side of (2) to zero and solving for $\bar{V}$ as follows:

$$
\bar{V}_{e q}=k_{v} \sqrt{\frac{\sigma_{\beta} \pm \sqrt{\sigma_{\beta}^{2}-6 \alpha\left(k_{i} / k_{v}\right)\left(C_{\alpha} \bar{P}_{e q}+S_{\alpha} \bar{Q}_{e q}\right)}}{3 \alpha}},
$$

where $\sigma_{\beta}=\left(\sigma-k_{v} k_{i} C_{\beta}\right)$. The $\bar{P}_{e q}$ and $\bar{Q}_{e q}$ denote the active and reactive power at the equilibrium. Both the roots of (4) are real valued if the following inequality holds:

$$
\left(C_{\alpha} \bar{P}+S_{\alpha} \bar{Q}\right)<S_{c r}:=\frac{\sigma_{\beta}^{2}}{6 \alpha\left(k_{i} / k_{v}\right)},
$$

where $k_{i}>0$ and $k_{v}>0 . S_{c r}$ denotes the critical power constant with corresponding critical value of the inverter RMS output voltage $\bar{V}_{c r}=k_{v} \sqrt{\frac{\sigma_{\beta}}{3 \alpha}}$. A local stability analysis of the high voltage solution of [4] (similar to [5]) is not considered. In the subsequent analysis, we assume that the high voltage solution of (4) is locally asymptotically stable and with a slight abuse of notation, we denote it by $\bar{V}_{e q}$. Using (4), the open circuit voltage $\bar{V}_{o c}=k_{v} \sqrt{\frac{2 \sigma_{\beta}}{3 \alpha}}$ for the VO-controlled inverter (i.e. $\bar{P}_{e q}=\bar{Q}_{e q}=0$ ).

2) Frequency Regulation Characteristics: Solving the phase angle dynamics (3) for equilibrium point gives the system's frequency $\omega_{e q}$ in steady-state as follows:

$$
\omega_{e q}=\omega^{*}+\frac{k_{v} k_{i}}{2 C}\left(\frac{C_{\alpha} \bar{Q}_{e q}}{\bar{V}_{e q}^{2}}-\frac{S_{\alpha} \bar{P}_{e q}}{\bar{V}_{e q}^{2}}-S_{\beta}\right),
$$

where $\bar{V}_{e q}$ is the high voltage solution of (4).

3) Dynamic Response: In order to quantify the dynamic response of the VO-controlled inverter, the time taken by the inverter to reach its open circuit voltage $\bar{V}_{o c}$ is considered. The following voltage dynamics of interest in a variable-separable ODE form are obtained from (2) by replacing $\bar{P}=\bar{Q}=0$ :

$$
\frac{d}{d t} \bar{V}=\frac{\sigma}{2 C}\left(\bar{V}-\frac{\beta}{2} \bar{V}^{3}\right)-\frac{k_{v} k_{i} C_{\beta}}{2 C} \bar{V} .
$$

The rise time $t_{\text {rise }}$ is the time taken by the inverter to buildup output voltage from $0.1 \bar{V}_{o c}$ to $0.9 \bar{V}_{o c}$. The $t_{\text {rise }} \approx \frac{6}{\omega^{*} \epsilon \sigma_{\beta}}$ is determined by separating variables in (7) and integrating under the limits from $0.1 \bar{V}_{o c}$ to $0.9 \bar{V}_{o c}$, where $\epsilon=\sqrt{L / C}$. 


\section{PI Controller Dynamics}

The PI controller dynamics used to regulate active power are $k_{v}=K_{P}^{p}\left(\bar{P}-P^{*}\right)+e_{p}$ where $\dot{e}_{p}=K_{I}^{p}\left(\bar{P}-P^{*}\right)$. Similarly, the PI controller dynamics used to regulate reactive power are $k_{i}=K_{P}^{q}\left(\bar{Q}-Q^{*}\right)+e_{q}$ where $\dot{e}_{q}=K_{I}^{q}\left(\bar{Q}-Q^{*}\right)$. The $e_{p}$ and $e_{q}$ are the PI controllers' states [9].

\section{VOC PARAmeter Design Procedure}

A parameter design procedure is presented for $\mathrm{VO}$ controlled inverters with current feedback after the LCL filter. The parameters are selected such that the VO-controlled inverter satisfy the desired ac-performance specifications.

\section{A. Designing the Scaling Factors}

The voltage scaling factor $k_{v}$ is chosen equal to $\bar{V}_{o c}$ to standardise the design procedure such that the virtual oscillator capacitor voltage is equal to $1 \mathrm{~V}$ RMS when the inverter's output voltage is equal to $\bar{V}_{o c}$. The current feedback gain $k_{i}$ is chosen as the ratio of $\bar{V}_{\min }$ to $\bar{S}_{\max }$. The $\bar{V}_{\min }$ corresponds to the constant $\bar{S}_{\max }$ defined by:

$$
\bar{S}_{\text {max }}=\max _{\bar{P}^{2}+\bar{Q}^{2} \leq\left|\bar{S}_{\text {rated }}\right|^{2}}\left(C_{\alpha} \bar{P}+S_{\alpha} \bar{Q}\right) .
$$

By choosing the gains as:

$$
k_{v}:=\bar{V}_{o c}, \quad k_{i}:=\frac{\bar{V}_{\min }}{\bar{S}_{\max }},
$$

the parallel-connected inverters in a system share the power proportional to their power ratings [5], [6].

\section{B. Designing the Voltage Regulation Parameters}

A design procedure for the virtual oscillator negative resistance element $R=\frac{-1}{\sigma}$ and the coefficient of nonlinear current source $\alpha$ is presented. The proposed design procedure ensures the RMS output voltage to stay within the range: $\bar{V}_{\text {min }} \leq \bar{V}_{e q} \leq \bar{V}_{o c}$ for $\bar{S}_{\max } \geq\left(C_{\alpha} \bar{P}_{e q}+S_{\alpha} \bar{Q}_{e q}\right) \geq 0$. The definition of $V_{O C}$ and the choice of $k_{v}$ in (9) results in $\sigma=\frac{3 \alpha}{2}+k_{i} k_{v} C_{\beta}$. Substituting $\left(C_{\alpha} \bar{P}_{e q}+S_{\alpha} \bar{Q}_{e q}\right)=\bar{S}_{\max }$, $\bar{V}_{e q}=\bar{V}_{\min }, k_{v}$ and $k_{i}$ as in (9), $\alpha=\frac{2}{3} \sigma_{\beta}$ and $\sigma_{\beta}=$ $\left(\sigma-k_{v} k_{i} C_{\beta}\right)$ in the high voltage solution of (4), we get:

$$
\sigma=\frac{\bar{V}_{o c}}{\bar{V}_{\min }} \frac{\bar{V}_{o c}^{2}}{\bar{V}_{o c}^{2}-\bar{V}_{\min }^{2}}+\frac{\bar{V}_{\min } \bar{V}_{o c} C_{\beta}}{\bar{S}_{\max }} .
$$

\section{Designing the Harmonic Oscillator Paramters}

In order to derive a set of constraints to determine the parameters $L$ and $C$, the frequency regulation characteristics (6), the rise time $t_{\text {rise }}$ and the ratio of the amplitude of the third harmonic to the fundamental [5, Eq. 41] are considered. While designing the harmonic oscillator parameters, one of the design input is the maximum permissible frequency deviation $|\Delta \omega|_{\max }$. Let us start with the frequency regulation characteristics in (6) and define the following constant:

$$
\bar{S}_{|\Delta \omega|_{\max }}=\max _{\bar{P}^{2}+\bar{Q}^{2} \leq\left|\bar{S}_{\text {rated }}\right|^{2}}\left(C_{\alpha} \bar{Q}-S_{\alpha} \bar{P}\right) .
$$

Using the worst-case operating condition for the output voltage (corresponding to $\bar{S}_{\max }$ that results in the minimum permissible voltage $\bar{V}_{\text {min }}$ ) and substituting the scaling factors from (9) into (6), the lower bound on capacitance $C$ is given by:

$$
C \geq \frac{1}{2|\Delta \omega|_{\max }}\left(\frac{\bar{S}_{|\Delta \omega|_{\max }} \bar{V}_{o c}}{\bar{V}_{\min } \bar{S}_{\max }}-\frac{S_{\beta} \bar{V}_{o c} \bar{V}_{\min }}{\bar{S}_{\max }}\right)=: C_{|\Delta \omega|_{\max }}^{\min } .
$$

Define the maximum permissible rise time $t_{\text {rise }}^{\max }$ as the design input. Now, considering the $t_{\text {rise }}$ of an unloaded inverter and (10), the upper bound on the capacitance $C$ is defined as:

$$
C \leq \frac{t_{r i s e}^{\max }}{6} \frac{\bar{V}_{o c}}{\bar{V}_{\min }} \frac{\bar{V}_{o c}^{2}}{\bar{V}_{o c}^{2}-\bar{V}_{\min }^{2}}=: C_{t_{\text {rise }}^{\max }}
$$

Finally, the third design input is the maximum-permissible ratio of the amplitude of third harmonic to the fundamental $\delta_{3: 1}^{\max }$ where $\delta_{3: 1}=\frac{\epsilon \sigma}{8}$ as defined in [5. Eq. 41]. Replacing (10) in the expression for $\delta_{3: 1}^{\max }$, we get another lower bound on the capacitance $C$ given by:

$C \geq\left(\frac{1}{8 \omega^{*} \delta_{3: 1}^{\max }}\right)\left(\frac{\bar{V}_{o c}}{\bar{V}_{\min }} \frac{\bar{V}_{o c}^{2}}{\bar{V}_{o c}^{2}-\bar{V}_{\min }^{2}}+\frac{\bar{V}_{\min } \bar{V}_{o c} C_{\beta}}{\bar{S}_{\max }}\right)=: C_{\delta_{3: 1}^{\min }}$.

The constraints $(12)-(14)$ define a permissible range for the capacitance satisfying the desired frequency regulation, rise time and harmonic distortion specifications. The permissible range of capacitance $C$ can be written as:

$$
\max \left\{C_{|\Delta \omega|_{\max }}^{\min }, C_{\delta_{3: 1}}^{\min }\right\} \leq C \leq C_{t_{\text {rise }}^{\max }} .
$$

Once the value is chosen for the capacitance $C$, the inductance can be determined as $L=\frac{1}{C\left(\omega^{*}\right)^{2}}$. Note that it may be possible (15) does not hold for the set of design inputs $\left\{|\Delta \omega|_{\max }, t_{\text {rise }}^{\max }, \delta_{3: 1}^{\max }\right\}$ and necessitates a design trade-off.

\section{POWER Dispatch}

The power dispatch technique presented in [9] is extended to the averaged VOC model with current feedback after the output LCL filter. Inverter 1 simultaneously regulates both the active and reactive power according to the desired power setpoint $\left(P_{1}^{*}, Q_{1}^{*}\right)$ using the PI controllers that continuously tune the VOC parameters $k_{v, 1}$ and $k_{i, 1}$ as in [9].

\section{A. Power Security Constraint}

An updated power security constraint is derived to determine the achievable power set-points and guarantee the existence of real valued control inputs.

Theorem 1. Assuming the averaged model of two VOC inverters with current feedback after the output LCL filter that synchronise to a common frequency, and are connected to a common fixed impedance load $z_{L}$ through line impedance values $z_{l, 1}$ and $z_{l, 2}$, respectively, the desired output power set-point $\bar{P}_{1}^{*}$ and $\bar{Q}_{1}^{*}$ for inverter 1 can be achieved and there exists corresponding real-valued current feedback gain $k_{i, 1}^{\iota}$ and voltage scaling factor $k_{v, 1}^{\iota}$, if the following security constraint is satisfied for the averaged VOC-dynamics:

$$
\sigma_{1} \bar{V}_{1}^{\iota 2}-\mu\left(C_{\alpha, 1} \bar{P}_{1}^{*}+S_{\alpha, 1} \bar{Q}_{1}^{*}+C_{\beta, 1} \bar{V}_{1}^{\iota 2}\right)>0,
$$

where,

$$
\mu=k_{v, 1}^{\iota} k_{i, 1}^{\iota}=k_{v, 2} k_{i, 2} \frac{\left(\frac{C_{\alpha, 2} \bar{Q}_{2}^{\iota}-S_{\alpha, 2} \bar{P}_{2}^{\iota}}{\bar{V}_{2}^{\iota 2}}-S_{\beta, 2}\right)}{\left(\frac{C_{\alpha, 1} \bar{Q}_{1}^{*}-S_{\alpha, 1} \bar{P}_{1}^{*}}{\bar{V}_{1}^{\iota 2}}-S_{\beta, 1}\right)} .
$$

$\bar{V}_{2}^{\iota}$ and $\bar{Q}_{2}^{\iota}$ denote the steady-state averaged output voltage magnitude and reactive power supplied by inverter 2 , respectively, corresponding to the desired power set-point $\left(\bar{P}_{1}^{*}, \bar{Q}_{1}^{*}\right)$.

The proof of this theorem is similar to [9, Theorem 1] and is omitted due to the space limitation. The updated control laws 
Table I

SYSTEM PARAMETERS

\begin{tabular}{llccc}
\hline \hline Symbol & Parameter & Inverter 1 & Inverter 2 & Unit \\
\hline$k_{i}$ & Current feedback gain & 0.15225 & 0.15225 & $\mathrm{~A} / \mathrm{A}$ \\
$k_{v}$ & Voltage scaling factor & 126 & 126 & $\mathrm{~V} / \mathrm{V}$ \\
$\sigma$ & Conductance & 6.09256 & 6.09256 & $\Omega^{-1}$ \\
$\alpha$ & Cubic-current source coefficient & 4.06184 & 4.06184 & $\mathrm{~A} / \mathrm{V}^{3}$ \\
$L$ & Oscillator inductance & 34.661 & 34.661 & $\mu \mathrm{H}$ \\
$C$ & Oscillator capacitance & 0.203 & 0.203 & $\mathrm{~F}$ \\
\hline
\end{tabular}

Table II

AC PERformance Specifications

\begin{tabular}{|c|c|c|c|}
\hline Symbol & Parameter & Value & Unit \\
\hline$\overline{\bar{V}}_{\text {oc }}$ & RMS open-circuit voltage & 126 & $\mathrm{~V}$ \\
\hline $\bar{V}_{\min }$ & RMS rated power voltage & 114 & $\mathrm{~V}$ \\
\hline $\bar{P}_{\text {rated }}$ & Rated real power & 750 & W \\
\hline$\left|\bar{Q}_{\text {rated }}\right|$ & Rated reactive power & 750 & VAr \\
\hline$\omega^{*}$ & Nominal oscillator frequency & $2 \pi 60$ & $\mathrm{rad} / \mathrm{s}$ \\
\hline$|\Delta \omega| \max$ & Maximum frequency offset & $2 \pi 0.5$ & $\mathrm{rad} / \mathrm{s}$ \\
\hline$t_{\text {rise }}^{\max }$ & Maximum rise time & 0.2 & $\mathrm{~s}$ \\
\hline$\delta_{3: 1}^{\max }$ & $3^{\text {rd }}$ to $1^{\text {st }}$ harmonic ratio & 1 & $\%$ \\
\hline
\end{tabular}

to determine the control inputs $k_{v, 1}^{\iota}$ and $k_{i, 1}^{\iota}$ corresponding to the desired power set-point $\left(\bar{P}_{1}^{*}, \bar{Q}_{1}^{*}\right)$ are:

$k_{v, 1}^{\iota}= \pm \sqrt{\frac{\left(\sigma_{1}-\mu C_{\beta, 1}\right) \bar{V}_{1}^{\iota 4}}{\left(\sigma_{1}-\mu C_{\beta, 1}\right) \bar{V}_{1}^{\iota 2}-\mu\left(C_{\alpha, 1} \bar{P}_{1}^{*}+S_{\alpha, 1} \bar{Q}_{1}^{*}\right)}}, k_{i, 1}^{\iota}=\frac{\mu}{k_{v, 1}^{\iota}}$.

\section{Simulation Results}

Simulation results in MATLAB (with ideal voltage sources) are presented for a number of scenarios to validate the proposed averaged model. The line, load and filter parameters $R_{f}=R_{l}=0.15 \Omega, L_{f}=L_{l}=2.48 \mathrm{mH}, R_{c}=3.3 \Omega$, $C_{f}=4.7 \mu \mathrm{F}, R_{g}=0.13 \Omega, L_{g}=0.97 \mathrm{mH}, R_{L}=22.1 \Omega$ and $L_{L}=14.4 \mathrm{mH}$. The system parameters and ac-performance specifications are as in Table II and Table II

\section{A. Embedded Droop Characteristics}

In order to validate the embedded droop characteristics (4), (6) within the averaged VOC dynamics, a comparison is made with the actual VOC dynamics (1) with current feedback after the LCL filter. In Fig. 2, it can be seen that the embedded droop characteristics are close to the actual VOC dynamics. In order to obtain the $\bar{V}-\bar{P}$ and $\omega-\bar{Q}$ droop characteristics, the LCL filter is assumed to be ideal (i.e. $R_{f}=R_{c}=R_{g}=0$ ) resulting in $S_{\alpha}=0$ and the parameters in Table I are rederived according to the design procedure in Section IV

\section{B. Rise Time and Harmonics Analysis}

In Fig. 3, the rise time and harmonics analysis is presented for an unloaded inverter. It can be seen that the VO-controlled inverter closely follows the design inputs $t_{\text {rise }}^{\max }$ and $\delta_{3: 1}^{\max }$, thus validating the parameter design procedure in Section IV

\section{Models Comparison}

In Fig. 4, a comparison is made between the previously reported averaged VOC model [5], the proposed averaged
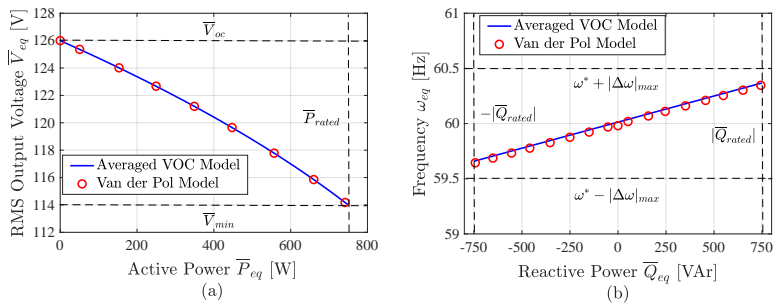

Figure 2. A comparison between the embedded droop characteristics within the averaged VOC dynamics [2)-(3) and actual VOC dynamics (1).
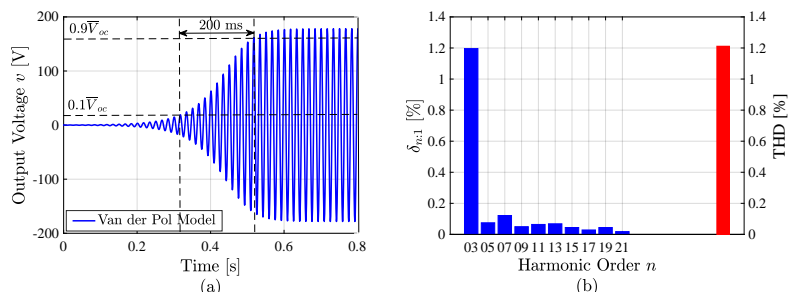

Figure 3. The rise time and harmonics analysis shows that the virtual oscillator controlled inverter closely follows the desired design inputs $t_{\text {rise }}^{\max }$ and $\delta_{3: 1}^{\max }$.

VOC model (2)-(3) and actual VOC dynamics (1). Note that the proposed averaged VOC model follows the actual VOC dynamics more closely as compared to the previously reported averaged VOC model. For comparison, the LCL filter is chosen with higher values of filter parameters $\left(L_{f}^{\iota}=10 L_{f}, C_{f}^{\iota}=\right.$ $\left.19.75 C_{f}\right)$ and the epsilon is chosen to be $\epsilon^{\iota}=\frac{\epsilon}{8}=\frac{\sqrt{L / C}}{8}$. The $z_{L}=6.9+j 16.6 \Omega$ with $z_{S}=44.24+j 10.85 \Omega$ inparallel to implement the step-up and step-down changes. The system parameters in Table $\mathrm{I}$ are re-derived according to the design procedure in Section IV Note that the difference between the previously reported averaged VOC model and actual VOC dynamics starts to increase for a higher value of filter capacitance. The output power is computed using a moving average window where window's length is updated based on the frequency recovered from actual VOC dynamics.

\section{Power Dispatch}

In Fig. 5, the power dispatch results are presented for a number of scenarios as listed in Table [II. Inverter 1 simultaneously regulates both the active and reactive power using the two PI controllers [9] that continuously tune the voltage scaling factor $k_{v, 1}$ and current feedback gain $k_{i, 1}$. The inverter 2 supplies the remaining power to the load acting like a slack bus. The PI controllers' proportional and integral gains are $K_{P}^{p}=-0.001, K_{I}^{p}=-0.15, K_{P}^{q}=0.0001$ and $K_{I}^{q}=0.01$. In Fig. 5 inverter 1 tracks the desired power set-points effectively while satisfying the security constraint (16). The corresponding changes in the inverter 2 power, load power, control inputs and voltages are also presented.

\section{CONCLUSION}

The LCL filter is considered an essential part of commercial inverters to filter out the harmonics and improve the output voltage. Keeping this in view, an averaged VOC model is derived for inverters with current feedback after the output LCL filter. The averaged model uncovers the embedded droopcharacteristics within the averaged VOC dynamics and simpli- 

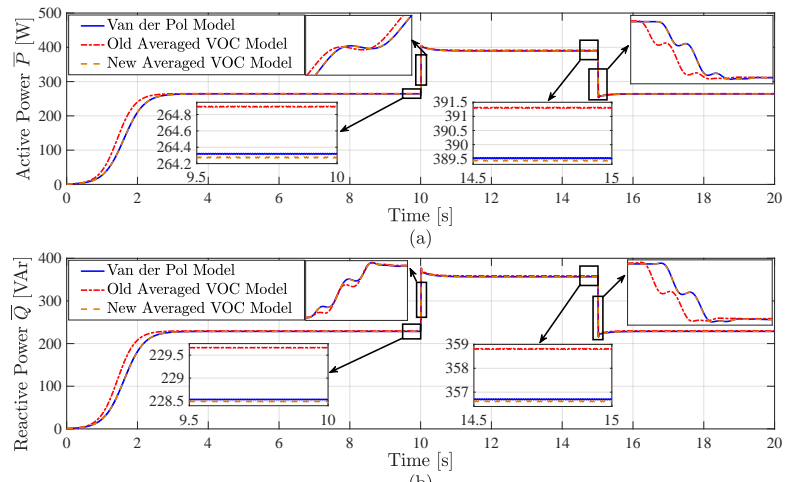

(b)
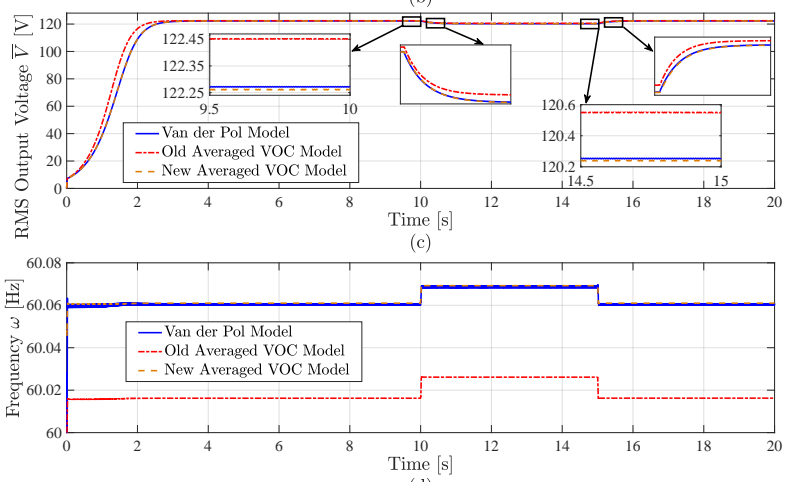

(d)

Figure 4. Comparison between previously reported averaged VOC model [5], the proposed averaged VOC model (2)-(3) and actual VOC dynamics (1): (a) active power, (b) reactive power, (c) voltages, (d) frequency.

Table III

POWER Dispatch SET-POINTS

\begin{tabular}{cccc}
\hline \hline Case No. & $P_{1}^{*}[\mathrm{~W}]$ & $Q_{1}^{*}[\mathrm{VAr}]$ & Time $[\mathrm{s}]$ \\
\hline 1 & - & - & $0-5$ \\
2 & 500 & 83 & $5-15$ \\
3 & 500 & 120 & $15-25$ \\
4 & 500 & 50 & $25-35$ \\
5 & 100 & 50 & $35-55$ \\
6 & 100 & 120 & $55-65$ \\
\hline \hline
\end{tabular}

fies the analysis. The voltage and frequency regulation characteristics are determined. Further, to enable the VO-controlled inverter to satisfy the desired ac-performance specifications, a parameter design procedure is presented. Moreover, a recently reported power dispatch technique is extended to the proposed averaged VOC model with current feedback after the output LCL filter. The updated control laws are derived to determine the control inputs corresponding to a particular power setpoint. An updated power security constraint is derived to determine the feasible operating region. The power security constraint is helpful in planning the optimal power flow in an electric grid. The simulation results validate the proposed averaged VOC model and power dispatch technique. Future work can include generalising the method to multiple inverters, stability analysis and experimental validation.

\section{APPENDIX}

A. Derivation of the Averaged VOC Model with an LCL Filter

Consider an inverter with current feedback after the output LCL filter as shown in Fig. 1. Let us denote a phasor associated

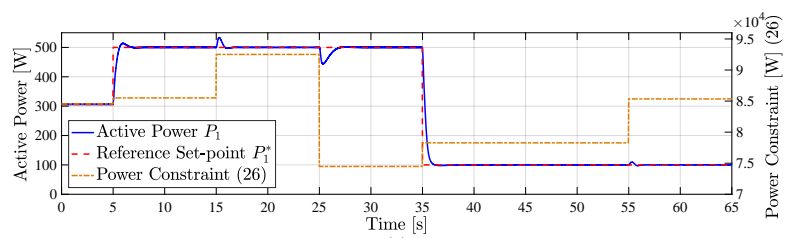

(a)
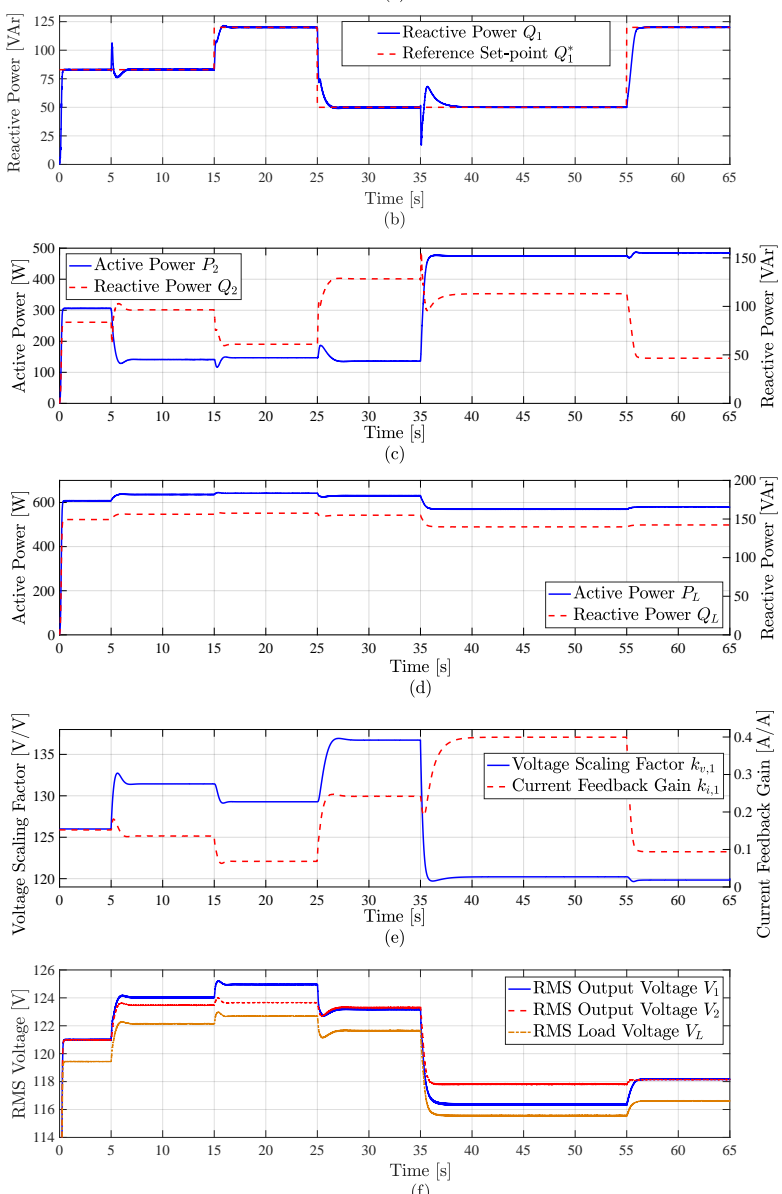

Figure 5. Power dispatch results for inverter 1 while inverter 2 supplies the remaining load power: (a) inverter 1 active power, power security constraint and reference set-point, (b) inverter 1 reactive power and reference set-point, (c) inverter 2 output power, (d) load power, (e) control inputs, (f) voltages.

with a time domain variable (.) by $\overrightarrow{(.)}$. The $\vec{V}$ denotes the voltage before the LCL filter, the $\vec{V}_{o}$ denotes the filter capacitor voltage and the $\vec{V}_{g}$ denotes the voltage after the LCL filter. Similarly, the $\overrightarrow{I_{f}}$ denotes the current flowing through filter inductor $L_{f}$, the $\overrightarrow{I_{c}}$ denotes the filter capacitor current and the $\overrightarrow{I_{g}}$ is the current flowing through filter inductor $L_{g}$. Solving the network equations, we have:

$$
\overrightarrow{I_{g}}=\left[\begin{array}{ll}
Z_{\alpha} \angle \theta_{\alpha} & Z_{\beta} \angle \theta_{\beta}
\end{array}\right]\left[\begin{array}{c}
\overrightarrow{I_{f}} \\
\vec{V}
\end{array}\right],
$$

where $z_{\alpha}=Z_{\alpha} \angle \theta_{\alpha}=\frac{z_{c}+z_{f}}{z_{c}}$ and $z_{\beta}=Z_{\beta} \angle \theta_{\beta}=\frac{-1}{z_{c}}$ are the impedance constants defined at $\omega^{*}$. Further, we have [5]:

$$
\frac{d}{d t} \phi=\omega^{*}+\frac{d}{d t} \theta^{*}=\omega+\frac{d}{d t} \theta,
$$

where the instantaneous phase angle is denoted by $\phi$, the nominal grid frequency is denoted by $\omega^{*}$ and the load dependent steady-state frequency is denoted by $\omega$. The angles 
$\theta^{*}$ and $\theta$ denote the phase offset with respect to $\omega^{*} t$ and $\omega t$, respectively. The inverter output voltage is given by:

$$
v(t)=\sqrt{2} V(t) \cos \left(\omega^{*} t+\theta^{*}(t)\right)
$$

The instantaneous active and reactive output power of inverter in terms of $v(t)$ and $i(t)$ is given by:

$$
P(t)=v(t) i(t), \quad Q(t)=v\left(t-\frac{\pi}{2}\right) i(t) .
$$

The averaged active and reactive power over an ac-cycle $\frac{2 \pi}{\omega^{*}}$ is defined as:

$$
\bar{P}(t)=\frac{\omega^{*}}{2 \pi} \int_{s=t}^{t+2 \pi / \omega^{*}} P(s) d s, \quad \bar{Q}(t)=\frac{\omega^{*}}{2 \pi} \int_{s=t}^{t+2 \pi / \omega^{*}} Q(s) d s .
$$

In order to derive the averaged dynamics (2)-(3), a change of variable is made from $t \rightarrow \tau=\omega^{*} t$ and $\theta^{*}(\tau) \stackrel{(2)}{=} \phi\left(\tau / \omega^{*}\right)-\tau$. Expressing the actual VOC dynamics (1) as a function of $\theta^{*}$, we have [5]:

$$
\begin{aligned}
\frac{d V}{d \tau} & =\frac{\epsilon}{\sqrt{2}}\left(\sigma g\left(\sqrt{2} V \cos \left(\tau+\theta^{*}\right)\right)-k_{v} k_{i} i\right) \cos \left(\tau+\theta^{*}\right) \\
\frac{d \theta^{*}}{d \tau} & =-\frac{\epsilon}{\sqrt{2} V}\left(\sigma g\left(\sqrt{2} V \cos \left(\tau+\theta^{*}\right)\right)-k_{v} k_{i} i\right) \sin \left(\tau+\theta^{*}\right) .
\end{aligned}
$$

The dynamics in (24) are $2 \pi$ periodic functions in $\tau$. The averaged dynamics in the quasi-harmonic limit $\epsilon \searrow 0$ (following [5. Eq. 12]) are given by:

$$
\begin{aligned}
{\left[\begin{array}{c}
\dot{\bar{V}} \\
\dot{\dot{\theta}}^{*}
\end{array}\right]=} & \frac{\epsilon \sigma}{2 \pi \sqrt{2}} \int_{0}^{2 \pi} g\left(\sqrt{2} \bar{V} \cos \left(\tau+\bar{\theta}^{*}\right)\right)\left[\begin{array}{c}
\cos \left(\tau+\bar{\theta}^{*}\right) \\
\frac{-1}{\bar{V}} \sin \left(\tau+\bar{\theta}^{*}\right)
\end{array}\right] d \tau \\
& -\frac{\epsilon k_{v} k_{i}}{2 \pi \sqrt{2}} \int_{0}^{2 \pi} i\left[\begin{array}{c}
\cos \left(\tau+\bar{\theta}^{*}\right) \\
\frac{-1}{\bar{V}} \sin \left(\tau+\bar{\theta}^{*}\right)
\end{array}\right] d \tau, \\
= & \frac{\epsilon \sigma}{2}\left[\begin{array}{c}
\bar{V}-\frac{\beta}{2} \bar{V}^{3} \\
0
\end{array}\right]-\frac{\epsilon k_{v} k_{i}}{2 \pi \sqrt{2}} \int_{0}^{2 \pi} i\left[\begin{array}{c}
\cos \left(\tau+\bar{\theta}^{*}\right) \\
\frac{-1}{\bar{V}} \sin \left(\tau+\bar{\theta}^{*}\right)
\end{array}\right] d \tau .
\end{aligned}
$$

Changing the coordinates from $\tau$ to $t$ in (25) and keeping the $\mathcal{O}(\epsilon)$ terms only, we have:

$$
\begin{aligned}
& \frac{d}{d t}\left[\begin{array}{c}
\bar{V} \\
\bar{\theta}^{*}
\end{array}\right]=\frac{\sigma}{2 C}\left[\begin{array}{c}
\bar{V}-\frac{\beta}{2} \bar{V}^{3} \\
0
\end{array}\right] \\
& -\frac{k_{v} k_{i} \omega^{*}}{2 \pi \sqrt{2} C} \int_{0}^{\frac{2 \pi}{\omega^{*}}} i(t)\left[\begin{array}{c}
\cos \left(\omega^{*} t+\bar{\theta}^{*}\right) \\
\frac{-1}{\bar{V}} \sin \left(\omega^{*} t+\bar{\theta}^{*}\right)
\end{array}\right] d t .
\end{aligned}
$$

Lets define the impedance constants $C_{\alpha}=Z_{\alpha} \cos \theta_{\alpha}, C_{\beta}=$ $Z_{\beta} \cos \theta_{\beta}, S_{\alpha}=Z_{\alpha} \sin \theta_{\alpha}$ and $S_{\beta}=Z_{\beta} \sin \theta_{\beta}$. The current $i=\sqrt{2} I \cos \left(\omega^{*} t+\theta_{i}^{*}\right)$ where $I$ is the RMS current magnitude and $\theta_{i}^{*}$ is the phase-offset with respect to $\omega^{*} t$. Let $\vec{I}_{g} \Re$ denotes the real part of the current $\overrightarrow{I_{g}}(19)$ and is given by:

$$
{\overrightarrow{I_{g}}}_{\Re}=\sqrt{2}\left(Z_{\alpha} I \cos \left(\omega^{*} t+\theta_{i}^{*}+\theta_{\alpha}\right)+Z_{\beta} V \cos \left(\omega^{*} t+\theta^{*}+\theta_{\beta}\right)\right) .
$$

In order to derive the averaged VOC model, the current $i=i_{g}$ is replaced by its real part $\vec{I}_{g}$ in 26 , we get:

$$
\begin{aligned}
& \frac{d}{d t}\left[\begin{array}{c}
\bar{V} \\
\bar{\theta}^{*}
\end{array}\right]=\frac{\sigma}{2 C}\left[\begin{array}{c}
\bar{V}-\frac{\beta}{2} \bar{V}^{3} \\
0
\end{array}\right]-\frac{k_{v} k_{i} \omega^{*}}{4 \pi C} \int_{0}^{\frac{2 \pi}{\omega^{*}}}[ \\
& \begin{array}{l}
\frac{Z_{\alpha}}{\bar{Z}_{\alpha}} \sqrt{2} V(t) \cos \left(\omega^{*} t+\theta^{*}\right) \sqrt{2} I(t) \cos \left(\omega^{*} t+\theta_{i}^{*}+\theta_{\alpha}\right)+ \\
\frac{-Z_{\alpha}}{\bar{V}^{2}} \sqrt{2} V(t) \sin \left(\omega^{*} t+\theta^{*}\right) \sqrt{2} I(t) \cos \left(\omega^{*} t+\theta^{*}+\theta_{\alpha}\right)-
\end{array} \\
& \left.\begin{array}{c}
2 Z_{\beta} \bar{V} \cos \left(\omega^{*} t+\theta^{*}+\theta_{\beta}\right) \cos \left(\omega^{*} t+\theta^{*}\right) \\
2 Z_{\beta} \cos \left(\omega^{*} t+\theta^{*}+\theta_{\beta}\right) \sin \left(\omega^{*} t+\theta^{*}\right)
\end{array}\right] d t .
\end{aligned}
$$

Using the trigonometric identities and definition of instantaneous active and reactive power (22), we get:

$$
\begin{aligned}
& \frac{d}{d t}\left[\begin{array}{c}
\bar{V} \\
\bar{\theta}^{*}
\end{array}\right]=\frac{\sigma}{2 C}\left[\begin{array}{c}
\bar{V}-\frac{\beta}{2} \bar{V}^{3} \\
0
\end{array}\right]-\frac{k_{v} k_{i} \omega^{*}}{4 \pi C} \int_{0}^{\frac{2 \pi}{\omega^{*}}}[ \\
& \left.\begin{array}{l}
\frac{1}{\bar{V}}\left(C_{\alpha} P(t)+S_{\alpha} Q(t)\right)+Z_{\beta} \bar{V}\left(\cos \theta_{\beta}+\cos \left(2 \omega^{*} t+2 \theta^{*}+\theta_{\beta}\right)\right) \\
\overline{\bar{V}^{2}}\left(C_{\alpha} Q(t)-S_{\alpha} P(t)\right)-Z_{\beta}\left(\sin \left(2 \omega^{*} t+2 \theta^{*}+\theta_{\beta}\right)-\sin \theta_{\beta}\right)
\end{array}\right] d t .
\end{aligned}
$$

The averaged VOC dynamics (2)-(3) are recovered from (29).

\section{ACKNOWLEDGMENT}

The authors acknowledge the kind support of A.W. Tyree Foundation, and Australian Research Council through Discovery Projects Funding Scheme under Project DP180103200.

\section{REFERENCES}

[1] K. De Brabandere, B. Bolsens, J. Van den Keybus, A. Woyte, J. Driesen, and R. Belmans, "A voltage and frequency droop control method for parallel inverters," IEEE Transactions on power electronics, vol. 22 , no. 4, pp. 1107-1115, 2007.

[2] Y. A.-R. I. Mohamed and E. F. El-Saadany, "Adaptive decentralized droop controller to preserve power sharing stability of paralleled inverters in distributed generation microgrids," IEEE Transactions on Power Electronics, vol. 23, no. 6, pp. 2806-2816, 2008.

[3] R. Teodorescu, F. Blaabjerg, and M. Liserre, "Proportional-resonant controllers. a new breed of controllers suitable for grid-connected voltage-source converters," Proc. Optim, vol. 3, pp. 9-14, 2004.

[4] B. Shoeiby, R. Davoodnezhad, D. Holmes, and B. McGrath, "Voltagefrequency control of an islanded microgrid using the intrinsic droop characteristics of resonant current regulators," in Energy Conversion Congress and Exposition (ECCE), 2014 IEEE, pp. 68-75, IEEE, 2014.

[5] B. B. Johnson, M. Sinha, N. G. Ainsworth, F. Dörfler, and S. V. Dhople, "Synthesizing virtual oscillators to control islanded inverters," IEEE Transactions on Power Electronics, vol. 31, no. 8, pp. 6002-6015, 2016.

[6] B. B. Johnson, S. V. Dhople, A. O. Hamadeh, and P. T. Krein "Synchronization of parallel single-phase inverters with virtual oscillator control," IEEE Transactions on Power Electronics, vol. 29, no. 11, pp. 6124-6138, 2014.

[7] M. Ali, A. Sahoo, H. Nurdin, J. Ravishankar, and J. Fletcher, "On the power sharing dynamics of parallel-connected virtual oscillatorcontrolled and droop-controlled inverters in an ac microgrid," in IECON 2019-45th Annual Conference of the IEEE Industrial Electronics Society, vol. 1, pp. 3931-3936, IEEE, 2019.

[8] M. Ali, H. I. Nurdin, and J. E. Fletcher, "Output power regulation of a virtual oscillator controlled inverter," in 2018 IEEE 18th International Power Electronics and Motion Control Conference (PEMC), pp. 10851090, IEEE, 2018.

[9] M. Ali, H. I. Nurdin, and J. E. Fletcher, "Simultaneous regulation of active and reactive output power of parallel-connected virtual oscillator controlled inverters," in IECON 2018-44th Annual Conference of the IEEE Industrial Electronics Society, pp. 4051-4056, IEEE, 2018.

[10] M. Ali, J. Li, L. Callegaro, H. I. Nurdin, and J. E. Fletcher, "Regulation of active and reactive power of a virtual oscillator controlled inverter," IET Generation, Transmission \& Distribution, vol. 14, no. 1, pp. 62-69, 2019.

[11] D. Groß, M. Colombino, J.-S. Brouillon, and F. Dörfler, "The effect of transmission-line dynamics on grid-forming dispatchable virtual oscillator control," IEEE Transactions on Control of Network Systems, 2019.

[12] D. Raisz, T. T. Thai, and A. Monti, "Power control of virtual oscillator controlled inverters in grid-connected mode," IEEE Transactions on Power Electronics, 2018.

[13] G.-S. Seo, M. Colombino, I. Subotic, B. Johnson, D. Groß, and F. Dörfler, "Dispatchable virtual oscillator control for decentralized inverter-dominated power systems: Analysis and experiments," in 2019 IEEE Applied Power Electronics Conference and Exposition (APEC), pp. 561-566, IEEE, 2019.

[14] M. Ali, H. I. Nurdin, and J. E. Fletcher, "Dispatchable virtual oscillator control for single-phase islanded inverters: Analysis and experiments," IEEE Transactions on Industrial Electronics, 2020. [Early Access] Online Available: doi: 10.1109/TIE.2020.2991996. 